\title{
PREVALENCIA DA LINFADENITE CASEOSA EM OVINOS E CAPRINOS DE MUNICÍPIOS DO ESTADO DO AMAZONAS E SENSIBILIDADE IN VITRO DE Corynebacterium pseudotuberculosis AOS ANTIMICROBIANOS
}

\author{
José Vicente Ferreira Neto ${ }^{1}$ \\ Rodrigo Antônio Torres Matos 2 \\ Felício Garino Júnior ${ }^{3}$ \\ José Allan Soares de Araujo ${ }^{4}$ \\ Karolline de Assis Pereira
}

\begin{abstract}
RESUMO
A linfadenite caseosa (LC) é uma enfermidade que acomete pequenos ruminantes, caracterizada clinicamente por lesões abscedantes em linfonodos, pele e órgãos, causada principalmente pela bactéria Corynebacterium pseudotuberculosis. O presente estudo investigou a prevalência da LC em criatórios de pequenos ruminantes do estado do Amazonas, bem como a sensibilidade microbiana in vitro de C. pseudotuberculosis aos antimicrobianos. Foi realizado exame clínico em 1.280 animais, dos quais 4,53\% (58/1.280) dos animais apresentavam abscessos e/ou cicatrizes na pele e/ou linfonodos. No perfil de sensibilidade in vitro aos antimicrobianos, $100 \%$ dos isolados de $C$. pseudotuberculosis obtidos de ovinos foram sensíveis à ampicilina (8/8), amoxicilina/ácido clavulânico (8/8), cefotaxima (8/8), ciprofloxacina (8/8), norfloxacino (8/8), sulfazotrim e tetraciclina (8/8). Nos isolados de C. pseudotuberculosis obtidos de caprinos, verificou-se $100 \%$ de sensibilidade in vitro diante da amoxicilina/ácido clavulânico (5/5), cefotaxima (5/5), ciprofloxacina (5/5) e norfloxacino (5/5). Ratifica-se a importância do primeiro isolamento do agente em pequenos ruminantes no estado, bem como o primeiro estudo epidemiológico na Região Norte, constatando que a enfermidade possui baixa prevalência em ovinos e caprinos no Amazonas.
\end{abstract}

Palavras-chave: Corynebacterium spp., lindadenite infecciosa, etiologia, pequenos ruminantes, sensibilidade microbiana in vitro, abscessos.

\section{PREVALENCE OF CASSEOUS LYMPHADENITIS IN SHEEP AND GOATS OF THE AMAZON STATE MUNICIPALITY AND IN VITRO SUSCEPTIBILITY PATTERN OF Corynebacterium pseudotuberculosis TO ANTIMICROBIALS}

\begin{abstract}
Caseous lymphadenitis (CL) is a disease that affects small ruminants, clinically characterized by abscessing lesions in lymph nodes, skin and organs, caused mainly by the bacterium Corynebacterium pseudotuberculosis. The present study investigated the prevalence of CS in small ruminant farms in the state of Amazonas, as well as the in vitro microbial sensitivity of C. pseudotuberculosis to antimicrobials. A clinical examination was carried out on 1,280 animals evaluated, of which $4.53 \%$ (58 / 1,280) of the animals had abscesses and / or scars on the skin and / or lymph nodes. In the in vitro sensitivity profile to antimicrobials, $100 \%$ of $C$.

\footnotetext{
${ }^{1}$ Instituto Nacional de Pesquisas da Amazônia, INPA, Manaus, Amazonas, Brasil - vicentemedvet@ hotmail.com

${ }^{2}$ Centro Universitário CESMAC, Maceió, Alagoas, Brasil.

${ }^{3}$ Animal VETLAB laboratório de análises clínicas veterinárias, Patos, Paraíba, Brasil.

${ }^{4}$ Escola Superior Batista do Amazonas, ESBAM, Manaus, Amazonas, Brasil.

${ }^{5}$ Médica Veterinária, Manaus, Amazonas, Brasil. karolassis.vet@gmail.com
}

Ferreira Neto JV, Matos RAT, Garino Junior F, Araújo JAS, Pereira KA. Prevalência da linfadenite caseosa em ovinos e caprinos de municípios do estado do amazonas e sensibilidade in vitro de Corynebacterium pseudotuberculosis aos antimicrobianos. Vet. e Zootec. 2020; 001-010.
\end{abstract}


pseudotuberculosis isolates obtained from sheep were sensitive to ampicillin (8/8), amoxicillin / clavulanic acid (8/8), cefotaxime (8/8), ciprofloxacin (8/8), norfloxacin (8/8), sulfazotrim and tetracycline (8/8). In isolates of $C$. pseudotuberculosis obtained from goats, there was $100 \%$ sensitivity in vitro to amoxicillin / clavulanic acid (5/5), cefotaxime (5/5), ciprofloxacin (5/5) and norfloxacin (5/5). The importance of the first isolation of the agent in small ruminants in the state is ratified, as well as the first epidemiological study in the North Region, confirming that the disease has a low prevalence in sheep and goats in Amazonas.

Key words: Corynebacterium spp., Infectious lindadenitis, etiology, small ruminants, in vitro microbial sensitivity, abscesses.

\section{PREVALENCIA DE LINFADENITIS CASEOSA EN OVINOS Y CAPRINOS DEL MUNICIPIO DEL ESTADO DE AMAZONA Y EN LA SUSCEPTIBILIDAD IN VITRO DE LA Corynebacterium pseudotuberculosis A ANTIMICROBIANOS}

\section{RESUMEN}

La linfadenitis caseosa (LC) es una enfermedad que afecta a pequeños rumiantes, caracterizada clínicamente por lesiones de absceso en los ganglios linfáticos, la piel y los órganos, causada principalmente por la bacteria Corynebacterium pseudotuberculosis. El presente estudio investigó la prevalencia de LC en pequeñas granjas de rumiantes en el estado de Amazona, así como la sensibilidad microbiana in vitro de C. pseudotuberculosis a los antimicrobianos. Se realizó un examen clínico en 1.280 animales evaluados, de los cuales $4.53 \%$ (58 / 1,280) de los animales tenían abscesos y / o cicatrices en la piel y / o ganglios linfáticos. En el perfil de sensibilidad in vitro a los antimicrobianos, el $100 \%$ de los aislados de $C$. pseudotuberculosis obtenidos de ovejas eran sensibles a ampicilina (8/8), amoxicilina / ácido clavulánico (8/8), cefotaxima (8/8), ciprofloxacina $(8 / 8)$, norfloxacino $(8 / 8)$, sulfazotrim y tetraciclina (8/8). En los aislados de $C$. pseudotuberculosis obtenidos de cabras, $100 \%$ de sensibilidad in vitro a amoxicilina / ácido clavulánico (5/5), cefotaxima (5/5), ciprofloxacina $(5 / 5)$ y norfloxacino (5/5). Se ratifica la importancia del primer aislamiento del agente en pequeños rumiantes en el estado, así como el primer estudio epidemiológico en la Región Norte, lo que confirma que la enfermedad tiene una baja prevalencia en ovejas y cabras en Amazona.

Palabras clave: Corynebacterium spp., Lindadenitis infecciosa, etiología, pequeños rumiantes, sensibilidad microbiana in vitro, abscesos.

\section{INTRODUÇÃO}

Em 2016, estimou-se que a caprinocultura e ovinocultura eram representadas por 1,02 bilhão e 1,1 bilhão de animais, respectivamente (1). O rebanho nacional de caprinos em 2014 alcançou 8,85 milhões de cabeças, das quais 8,1 milhões concentradas na Região Nordeste, enquanto o rebanho ovino foi estimado em 2014 em 17,6 milhões de cabeças, predominantemente nas regiões Nordeste com 10,1 milhões de ovinos e Sul com 5,1 milhões de animais (2).

De acordo com dados do IBGE (3), o estado do Amazonas possuía rebanho de 21.488 caprinos e 69.131 ovinos. Os maiores efetivos de caprinos e ovinos do Brasil estão nas regiões nordeste e sul. Em contraste, a região norte possui um dos menores efetivos, embora recentemente estejam sendo realizados investimentos para aquisição de animais visando 
aumentar os rebanhos da região. Atualmente, o rebanho de ovinos e caprinos no Amazonas soma 65 mil cabeças, das quais mais de $80 \%$ são ovinos (4).

A produção de pequenos ruminantes no estado do Amazonas não atende à demanda local e boa parte da carne consumida é adquirida de outros estados brasileiros (4).

A linfadenite caseosa (LC) é uma enfermidade infectocontagiosa de evolução crônica, que acomete caprinos e ovinos. O agente etiológico da doença é a bactéria Corynebacterium pseudotuberculosis, que possui dois biovares, ovis e equi. O biovar ovis infecta ovinos e caprinos, enquanto o biovar equi acomete equinos, bovinos, camelos e búfalos $(5,6)$.

C. pseudotuberculosis são micro-organismos pleomórficos, Gram-positivos, não esporulados, aeróbicos, caracterizados pela formação de abscessos em linfonodos, tecido cutâneo e, ocasionalmente, em órgãos viscerais $(7,8,9)$.

A doença é endêmica no Brasil, e tem prevalência clínica que varia de $5 \%$ até $50 \%$, sendo mais comum em caprinos e ovinos deslanados (9). Diferentes estudos têm relatado a prevalência de LC em regiões e estados brasileiros, destacando-se na região nordeste (9-14), centro-oeste, região sudeste $(15-18)$ e região sul $(19,20)$. No entanto, na literatura consultada, não foi encontrado nenhum estudo de prevalência da doença na região Norte do Brasil.

Apesar de $C$. pseudotuberculosis apresentar boa sensibilidade in vitro aos antimicrobianos, o agente é habitualmente resistente ao tratamento in vivo, em virtude da presença intracelular do agente (resiste à fagocitose) e a formação de piogranulomas, que dificultam a ação dos antimicrobianos no foco infeccioso (21).

Neste cenário, o presente estudo investigou a prevalência da LC em ovinos e caprinos provenientes de municípios do estado do Amazonas, bem como o perfil de sensibilidade microbiana in vitro aos antimicrobianos dos isolados de C. pseudotuberculosis.

\section{MATERIAL E MÉTODOS}

\section{Comitê de Ética e Uso de Animais}

Protocolo sob o número 027/2016, estando de acordo com os princípios éticos de experimentação animal da "comissão de Ética no uso de animais" da Escola Superior Batista do Amazonas (ESBAM).

\section{Caracterização da área de estudo}

O estudo foi conduzido a partir de visitas técnicas em 19 propriedades criadoras de ovinos e caprinos, mais conhecidas na região, próximas à cidade de Manaus, responsáveis pelo abastecimento de carne, leite e seus derivados na capital Manauara, denominadas respectivamente, como propriedades A, B, C, D, E, F, G, H, I, J, K, L, M, N, O, P, Q, R, S. As propriedades utilizadas estão localizadas nos municípios de Manaus (Latitude: -3.10719, Longitude: -60.0261 $3^{\circ} 6^{\prime} 26^{\prime \prime} \mathrm{Sul}, 60^{\circ} 1^{\prime} 34^{\prime \prime}$ Oeste), Presidente Figueiredo (Latitude: 2.03435, Longitude: -60.0259 $2^{\circ} 2^{\prime} 4^{\prime \prime} \mathrm{Sul}, 60^{\circ} 1^{\prime} 33^{\prime \prime}$ Oeste), Rio Preto da Eva (Latitude: -

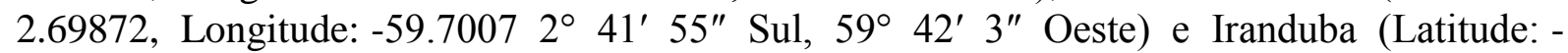
3.20113, Longitude: $-60.17823^{\circ} 12^{\prime} 4^{\prime \prime} \mathrm{Sul}, 60^{\circ} 10^{\prime} 42^{\prime \prime}$ Oeste), e os exames clínicos dos animais e colheitas de amostras foram realizados entre julho e agosto de 2016.

\section{Animais e colheita de amostras}

Os animais eram criados em sistemas intensivo e semi-intensivo. Foram avaliados clinicamente, segundo Feitosa (22), por conveniência, totalizando 1.280 animais. O número de caprinos e ovinos variou de 2 a 442 animais por propriedade. 
Os caprinos eram das raças Boer, Cariri, Anglo Nubiana, Saanen, Toggenburg, Pardo Alpina e sem raça definida (mestiços), enquanto os ovinos das raças Santa Inês, Dorper, Morada Nova e sem raça definida (mestiços).

Para avaliação da prevalência de ovinos e caprinos, todos os animais dos rebanhos foram inspecionados e, em seguida, identificados os que apresentavam aumento de volume nos linfonodos superficiais ou palpáveis e cicatrizes sugestivas de LC.

Para colheita de material, foi adotado como critério de inclusão das amostras abscessos que estavam "maduros" e com região central alopécica. Não foram colhidas amostras de animais sem os sinais supracitados. Adotando este critério, foram colhidas 13 amostras em 10 dos 1.280 animais avaliados. Em apenas um caprino foi possível identificar três abscessos aptos para a coleta e um ovino com dois abscessos. Nas áreas que apresentavam aumento de volume, em que o abscesso se encontrava flutuante, era realizada a tricotomia, antissepsia com solução de iodo a 10\%. Em seguida, com o auxílio de uma lâmina de bisturi esterilizada foi realizada uma incisão na região central do abscesso e colhido o conteúdo caseoso em tubos do tipo falcon esterilizados (Falcon ${ }^{\circledR}$, Nova York, Estado Unidos).

As amostras foram armazenadas em caixa isotérmica, conservadas em freezers a $-20^{\circ} \mathrm{C}$ no Instituto Leônidas \& Maria Deane - Fundação Oswaldo Cruz (ILMD/FIOCRUZ-AM) e, posteriormente, encaminhadas ao Laboratório de Microbiologia do Instituto Nacional de Ciência e Tecnologia (INCT), situado no Hospital Veterinário da Universidade Federal de Campina Grande-CSTR, Campus de Patos, para a realização dos exames microbiológicos.

\section{Análise microbiológica}

As amostras foram semeadas em placas contendo o meio de cultura ágar suplementado com sangue ovino desfibrinado a $5 \%$ (Himedia $^{\circledR}$, Bhaveshwar - Mumbai, Índia) e tubos contendo caldo BHI (Himedia ${ }^{\circledR}$, Bhaveshwar - Mumbai, Índia). Posteriormente, as placas e tubos foram incubados em condições de aerobiose a $37^{\circ} \mathrm{C}$ em estufa bacteriológica, e realizadas leituras a cada 24 horas, por três dias.

A identificação de $C$. pseudotuberculosis foi realizada com base em características macroscópicas e morfotintoriais (características das colônias e coloração de Gram), e provas bioquímicas, e.g., produção de urease, redução de nitrato, hidrólise de esculina, oxidação e fermentação (OF), Camp Test e fermentação de açúcares, glicose, sacarose, maltose, xilose, lactose e manitol (23).

\section{Teste de sensibilidade microbiana in vitro dos isolados aos antimicrobianos}

Para o teste de sensibilidade microbiana in vitro dos isolados de C. pseudotuberculosis aos antimicrobianos, foi utilizado o teste de difusão com discos Kirby-Bauer (24). Foram utilizados discos impregnados com os seguintes antimicrobianos: ampicilina $10 \mu \mathrm{g}$ (AMP), amoxicilina com ácido clavulânico $30 \mu \mathrm{g}$ (AMC), cefotaxima $30 \mu \mathrm{g}$ (CTX), ceftazidima 30 $\mu \mathrm{g}$ (CAZ), ciprofloxacina $05 \mu \mathrm{g}$ (CIP), gentamicina $10 \mu \mathrm{g}$ (GEN), norfloxacino $10 \mu \mathrm{g}$ (NOR), sulfametoxazole/trimetoprim $25 \mu \mathrm{g}$ (SUT) e tetraciclina $30 \mu \mathrm{g}$ (TET) (25). Para o teste de controle de qualidade foram utilizadas cepas de Staphylococcus aureus ATCC 25923 e Corynebacterium pseudotuberculosis INSQS 00212.

\section{Análise estatística}

Para comparação entre caprinos e ovinos quanto à prevalência de $\mathrm{LC}$, bem como a comparação entre fêmeas e machos, foi utilizado o teste de Fisher (26). Foi adotado o nível de 
significância de $5 \%$ e as análises foram realizadas com o programa estatístico SPSS for Windows versão 22.

\section{RESULTADOS}

Dos 1.280 animais avaliados 4,53\% (58/1.280) apresentaram evidências clínicas de LC (48 ovinos e 10 caprinos). Em 36,2 \% (21/58) destes animais haviam apenas cicatrizes de abscessos. Em 63,8\% (37/58) dos animais, os abscessos estavam intactos (Tabela 1).

Tabela 1. Frequências absolutas e relativas de lesões de LC em caprinos e ovinos criados em municípios do estado do Amazonas. Julho a agosto de 2016.

\begin{tabular}{cccc}
\hline Animais & $\begin{array}{c}\text { com linfadenite } \\
\text { caseosa }\end{array}$ & $\begin{array}{c}\text { sem linfadenite } \\
\text { caseosa }\end{array}$ & Total \\
\hline Ovinos & $48(5,19 \%)$ & $877(94,81 \%)$ & $925(100 \%)$ \\
Caprinos & $10(2,82 \%)$ & $345(97,18 \%)$ & $355(100 \%)$ \\
Total & 58 & 1243 & 1280 \\
\hline
\end{tabular}

Do total de ovinos com lesões clínicas compatíveis com LC, cinco $(5 / 48=10,4 \%)$ eram machos e quarenta e três $(43 / 48=89,6 \%)$ fêmeas. Do total de caprinos com lesões clínicas compatíveis com LC, quatro $(4 / 10=40 \%)$ eram machos e seis $(6 / 10=60 \%)$ fêmeas. Em relação ao número de ovinos e caprinos com evidências clínicas e ao sexo dos animais, houve diferença estatisticamente significante na comparação entre as duas espécies e entre machos e fêmeas $(p=0.0387)$. Os ovinos e caprinos com evidências clínicas da doença apresentavam aumento de volume nos linfonodos superficiais.

Os linfonodos mais acometidos em ovinos foram os linfonodos pré-escapulares $(12 / 36=33,33 \%)$, submandibulares $(6 / 36=16,66)$ e pré-crurais $(4 / 36=11,11 \%)$. Os linfonodos ou locais mais afetados em caprinos foram a região do peito $(3 / 10=30 \%)$, linfonodos submandibulares $(2 / 10=20 \%)$ e pré-crurais $(2 / 10=20 \%)$. Na tabela abaixo estão representados o número de linfonodos e locais mais afetados pela LC nas duas espécies (Tabela 2).

Das 13 amostras coletadas foi realizado o isolamento de C. pseudotuberculosis. Estas amostras foram coletadas de 10 animais ( 7 ovinos e 3 caprinos) que tinham sinais clínicos evidentes da doença. C. pseudotuberculosis revelou no meio de ágar sangue ovino desfibrinado (5\%), colônias pequenas, hemolíticas, esbranquiçadas e secas que não se aderiam ao meio.

No teste de sensibilidade in vitro aos antimicrobianos foi verificado em ovinos que $C$. pseudotuberculosis apresentou $100 \%$ de sensibilidade aos antimicrobianos ampicilina, amoxicilina/ácido clavulânico, cefotaxima, ciprofloxacino, norfloxacino, sulfametoxazole/trimetoprim, e tetraciclina. Em contraste, os isolados apresentaram elevada resistência à gentamicina (100\%) e a ceftazidima $(57,14 \%)$.

Em caprinos verificou-se que C. pseudotuberculosis apresentou $100 \%$ de sensibilidade aos antimicrobianos amoxicilina/ácido clavulânico, cefotaxima, ciprofloxacina, norfloxacino, bem como a ampicilina $(83,33 \%)$ e tetraciclina $(83,33 \%)$. Apresentou elevada resistência à ceftazidima $83,33 \%$ e à gentamicina e à sulfazotrim $50 \%$ (Tabela 3 ). 
Tabela 2. Distribuição dos linfonodos e locais mais comumente afetados por $C$. pseudotuberculosis em 30 ovinos positivos e sete caprinos positivos entre 1.280 animais clinicamente avaliados, provenientes propriedades rurais do estado do Amazonas. Julho a agosto de 2016.

\begin{tabular}{ccccc}
\hline Local (linfonodos) & $\begin{array}{c}\mathbf{N}^{\mathbf{0}} \text { de } \\
\text { linfonodos } \\
\text { caprinos }\end{array}$ & $\begin{array}{c}\text { Caprinos } \\
\mathbf{( \% )}\end{array}$ & $\begin{array}{c}\mathbf{N}^{\mathbf{0}} \text { de } \\
\text { linfonodos } \\
\text { ovinos }\end{array}$ & $\begin{array}{c}\text { Ovinos } \\
\mathbf{( \% )}\end{array}$ \\
\hline Pré-crural & 2 & 20 & 4 & 11,11 \\
Parotídeo & 0 & 0 & 2 & 5,55 \\
Submandibular & 2 & 20 & 6 & 16,66 \\
Abscesso no peito & 3 & 30 & 2 & 5,55 \\
Glândula mamária & 1 & 10 & 1 & 2,77 \\
Pré-escapular & 1 & 10 & 12 & 33,33 \\
Esterno & 0 & 0 & 3 & 8,33 \\
Garupa & 0 & 0 & 3 & 8,33 \\
Poplíteo & 0 & 0 & 1 & 2,77 \\
Pescoço & 1 & 10 & 1 & 2,77 \\
Escroto & 0 & 0 & 1 & 2,77 \\
Total & $\mathbf{1 0}$ & $\mathbf{1 0 0}$ & $\mathbf{3 6}$ & $\mathbf{1 0 0}$ \\
\hline
\end{tabular}

Tabela 3. Perfil de sensibilidade in vitro de 13 isolados de C. pseudotuberculosis obtidos de abscessos de ovinos e caprinos de municípios do estado do Amazonas. Julho a agosto de 2016.

\begin{tabular}{|c|c|c|c|c|}
\hline & \multicolumn{2}{|c|}{ Ovinos } & \multicolumn{2}{|c|}{ Caprinos } \\
\hline Antimicrobiano & $\begin{array}{c}\text { Sensível } \\
\%\end{array}$ & $\begin{array}{c}\text { Resistente } \\
\%\end{array}$ & $\begin{array}{c}\text { Sensível } \\
\%\end{array}$ & $\begin{array}{c}\text { Resistente } \\
\%\end{array}$ \\
\hline AMP & 100 & 0 & 83,33 & 16,67 \\
\hline $\mathrm{AMC}$ & 100 & 0 & 100 & 0 \\
\hline CTX & 100 & 0 & 100 & 0 \\
\hline CAZ & 14,29 & 57,14 & 0 & 83,33 \\
\hline CIP & 100 & 0 & 100 & 0 \\
\hline GEN & 0 & 100 & 50 & 50 \\
\hline NOR & 100 & 0 & 100 & 0 \\
\hline SUT & 100 & 0 & 50 & 50 \\
\hline TET & 100 & 0 & 83,33 & 16,67 \\
\hline
\end{tabular}

AMP $10 \mu \mathrm{g}$ - ampicilina; AMC $30 \mu \mathrm{g}$ - amoxicilina com ácido clavulânico; CTX $30 \mu \mathrm{g}$ - cefotaxima; CAZ 30 $\mu \mathrm{g}$ - ceftazidima; CIP $05 \mu \mathrm{g}$ - ciprofloxacina; GEN $10 \mu \mathrm{g}$ - gentamicina; NOR $10 \mu \mathrm{g}$ - norfloxacino; SUT 25 $\mu \mathrm{g}$ - sulfazotrin; TET $30 \mu \mathrm{g}$ - tetraciclina.

\section{DISCUSSÃO}

Verificou-se com o presente estudo que a LC tem prevalência menor na região amazônica quando comparada com outras regiões do país.

Estudos epidemiológicos têm demonstrado alta positividade dos animais na região nordeste $(11,27)$, resultado que discorda do obtido no presente estudo. Este fato pode estar relacionado com a variação no sistema de manejo de cada propriedade e maior presença de plantas espinhosas na pastagem.

A ocorrência de animais infectados nos rebanhos de ovinos e caprinos na região estudada, pode ser creditada às deficiências de medidas de controle sistemático nas fazendas, 
assim como a falta de controle no transporte e comercialização de animais, o que possibilita que animais infectados sejam introduzidos nos rebanhos, conforme relatado por Souza et al. (9).

Em estudo realizado por Andrade et al. (28) com caprinos e ovinos foi observado que de 640 animais examinados, 7,7\% (49,640) apresentavam evidências clínicas de LC, caracterizada por cicatrizes e/ou abscessos, de modo similar ao observado no presente estudo.

Quando comparados estatisticamente o número de caprinos e ovinos com evidências clínicas e o sexo desses animais, verificou-se diferença significante. Tal fato pode ser atribuído à distribuição dos animais entre as propriedades, sendo encontrado número maior de ovinos em relação ao de caprinos, bem como prevalecendo maior número de fêmeas nas propriedades estudadas. Andrade et al. (28) identificaram que deixar o abscesso romper naturalmente está associado a maior risco de ocorrência da infecção. Tal predisposição pode estar relacionada ao contato direto e indireto com o agente, nas pastagens, instalações e utensílios contaminados com as secreções purulentas dos abscessos, favorecendo a infecção pelo patógeno.

Considerando a localização dos abscessos nas duas espécies, observou-se que os linfonodos mais acometidos foram os pré-escapulares, submandibulares e pré-crurais. Estes resultados são semelhantes aos encontrados por Andrade et al. (28) e, provavelmente, a maior ocorrência nos linfonodos superficiais encontra reflexo na maior exposição dos animais a traumatismos, como em cercas de arame farpado, ou pela ingestão de alimentos grosseiros, ressecados ou pontiagudos (21).

A sensibilidade de $100 \%$ dos isolados observada para norfloxacino, assim como a sensibilidade de C. pseudotuberculosis abaixo de $60 \%$ para gentamicina, são resultados similares aos obtidos por Abreu et al. (29), que estudando LC em ovinos e caprinos no Sertão Pernambucano, encontraram sensibilidade dos isolados para norfloxacino de $93,5 \%$ e $32,2 \%$ para gentamicina.

Foi encontrada elevada sensibilidade dos isolados aos demais antimicrobianos, isso se deve ao fato de que os proprietários não fazem uso habitual de antibióticos na criação de caprinos e ovinos.

No presente estudo foram utilizadas diferentes classes de antimicrobianos, com indicação para $C$. pseudotuberculosis. Embora em alguns casos seja recomendado o tratamento, principalmente quando o animal tem elevado valor zootécnico, muitas vezes o mesmo não é eficaz, pois esta bactéria induz a formação de piogranulomas que limitam a ação dos fármacos no foco infeccioso $(8,21)$. Portanto, em muitos casos recomenda-se outras alternativas de tratamento ou mesmo o descarte dos animais.

A ausência de estudos epidemiológicos na região Norte, relativos às medidas de frequência da doença e a falta de laboratório de microbiologia veterinária para analises, são fatores limitantes ao estudo.

\section{CONCLUSÃO}

A LC está presente na ovinocaprinocultura amazonense. Portanto, ressalta-se a importância da realização de monitoramento para identificar os animais infectados nas propriedades, além de instituir medidas de controle para combater os avanços da doença.

Há a necessidade de conscientização dos criadores de ovinos e caprinos sobre a importância desta enfermidade e da realização de boas práticas de manejo para evitar a disseminação da doença entre os rebanhos.

\section{AGRADECIMENTOS}

Ferreira Neto JV, Matos RAT, Garino Junior F, Araújo JAS, Pereira KA. Prevalência da linfadenite caseosa em ovinos e caprinos de municípios do estado do amazonas e sensibilidade in vitro de Corynebacterium pseudotuberculosis aos antimicrobianos. Vet. e Zootec. 2020; 001-010. 
O autor agradece aos produtores rurais pela colaboração no desenvolvimento do trabalho e ao Laboratório de Microbiologia do Instituto Nacional de Ciência e Tecnologia (INCT) - UFCG - Campus de Patos - PB.

\section{REFERÊNCIAS}

1. Food and Agriculture Organization of the United Nations-FAO [Internet]. Rome: FAO; 2016 [cited 2016 Nov 18]. Available from: http://faostat3.fao.org/ download/Q/QA/E

2. Martins EC, Magalhães KA, Souza JDF, Guimarães VP, Barbosa CMP, Holanda Filho ZF. Cenários mundial e nacional da caprinocultura e da ovinocultura. Ativos Ovinos Caprinos. 2016;3(2):3-6.

3. Instituto Brasileiro de Geografia e Estatística-IBGE. Pecuária 2011 [Internet]. Rio de Janeiro: IBGE; 2011 [cited 2016 Oct 15]. Available from: http://www.ibge.gov.br/estadosat/temas.php?sigla =am\&tema=pecuaria2011

4. Instituto de Desenvolvimento Agropecuário e Florestal Sustentável do Estado do Amazonas-IDAM. Governo do Amazonas investe R\$ 6 milhões para estimular ovinocaprinocultura no Estado [Internet]. Manaus: IDAM; 2016 [cited 2016 Sept 29]. Available from: http://www.idam.am.gov.br/governo-do-amazonas-investe-r-6-milhoespara-estimular-ovinocaprinocultura-no-estado/

5. Dorella FA, Pacheco LG, Oliveira SC, Miyoshi A, Azevedo V. Corynebacterium pseudotuberculosis: microbiology, biochemical properties, pathogenesis and molecular studies of virulence. Vet Res. 2006;37(2):201-18.

6. Dorneles EM, Santana JA, Ribeiro D, Dorella FA, Guimarães AS, Moawad MS, et al. Evaluation of ERIC-PCR as genotyping method for Corynebacterium pseudotuberculosis isolates. PLoS One. 2014;9(6):e98758.

7. Alves FSF, Santiago LB, Pinheiro RR. Linfadenite caseosa: o estado da arte. Sobral: Embrapa Caprinos; 2007.

8. Fontaine MC, Baird GJ. Caseous lymphadenitis. Small Rumin Res. 2008;76:42-8.

9. Souza MF, Carvalho AQ, Garino Júnior F, Riet-Correa F. Linfadenite caseosa em ovinos deslanados abatidos em um frigorífico da Paraíba. Pesqui Vet Bras. 2011;31(3):224-30.

10. Andrade JSL. Linfadenite caseosa em ovinos e caprinos criados nas microrregiões de Piancó e Itaporanga - PB: inquérito e fatores de risco associados à doença. [dissertação] Patos: Universidade Federal de Campina Grande; 2007.

11. Carmo FB, Guimarães AS, Pauletti RB, Lage AP, Gonçalves VSP, Meyer R, et al. Prevalência de anticorpos contra a linfadenite caseosa em criações comerciais de ovinos no Distrito Federal, Brasil. Arq Inst Biol. 2012;79(2):293-6.

12. Martins RJ, Veschi JLA, Landim MAS, Carmo FB, Azevedo V, Miyoshi A, et al. Avaliação da presença de anticorpos anti-Corynebacterium pseudotuberculosis em ovinos do Município de Dormentes, PE. In: Anais da 6a Jornada de Iniciação Científica 
da Embrapa Semiárido; 2011; Petrolina, Pernambuco. Petrolina: Embrapa Seminário; 2011. p.397-403.

13. Silva RAB, Batista MCS, Nascimento CB, Alves RPA, Alves FSF, Pinheiro RR, et al. Caracterização zoosanitária da ovinocultura e da caprinocultura na microrregião homogênea de Teresina, Piauí, Brasil. Arq Inst Biol. 2011;78(4):593-8.

14. Teixeira WC, Santos HP, Silva JCR, Rizzo H, Marvulo MFV, Castro RS. Perfil zoosanitário dos rebanhos caprinos e ovinos em três mesorregiões do estado do Maranhão, Brasil. Acta Vet Bras. 2015;9(1):34-42.

15. Langenegger J, Langenegger $\mathrm{CH}$, Scherer PO. Prevalência e diagnóstico comparativo da linfadenite caseosa em caprinos do Estado do Rio de Janeiro. Pesqui Vet Bras. 1991;11(1/2):31-4.

16. Yorinori EH. Características dos sistemas de produção de pequenos ruminantes e prevalências da artrite-encefalite caprina (CAE) e MaediVisna (MV) ovina, nas regiões norte e nordeste de Minas Gerais [dissertação]. Belo Horizonte: Escola de Veterinária, Universidade Federal de Minas Gerais; 2001.

17. Guimarães AS, Seyffert N, Bastos BL, Portela RWD, Meyer R, Carmo FB, et al. Caseous lymphadenitis in sheep flocks of the state of Minas Gerais, Brazil: prevalence and management surveys. Small Rumin Res. 2009;87:86-91.

18. Seyffert N, Guimarães AS, Pacheco LGC, Portela RW, Bastos BL, Dorella FA, et al. High seroprevalence of caseous lymphadenitis in Brazilian goat herds revealed by Corynebacterium pseudotuberculosis secreted proteins-based ELISA. Res Vet Sci. 2010;88(1):50-5.

19. Silva MUD, Silva AEDF. Linfadenite caseosa em caprinos: observações de dois anos. In: Anais do 18o Congresso Brasileiro de Medicina Veterinária; 1982; Camboriú. Camboriú: Sociedade Brasileira de Medicina Veterinária; 1982. p.49-50.

20. Machado G, Gressler LT, Kirinus JK, Herrmann GP. Linfadenite caseosa em ovinos abatidos sob inspeção federal no estado do Rio Grande do Sul-estimativas de perdas. Acta Sci Vet. 2011;39(2):967.

21 - Megid J, Ribeiro MG, Paes ACP. Doenças infecciosas em animais de produção e de companhia. In: Ribeiro MG, Mota RA. Linfadenite caseosa ovina e caprina. Rio de Janeiro: Roca; 2016. p.388-98.

22. Feitosa FLF. Semiologia veterinária: a arte do diagnóstico. In: Feitosa FLF. Exame físico geral ou de rotina. 3a ed. São Paulo: Roca; 2014. p.51-68.

23. Quinn PJ, Markey BK, Leonard FC, FitzPatrick ES, Fanning S, Hartigan PJ. Veterinary microbiology and microbial disease. 2a ed. Oxford: Wiley-Blackwell; 2011.

24. Bauer AW, Kirby WMM, Sherris JC, Turck M. Antibiotic susceptibility testing by a standardized disk method. Am J Clin Pathol. 1966;45(4):493-6. 
25- Clinical and Laboratory Standars Institute-CLSI. Performance standards for antimicrobial susceptibility testing: twenty-fifth informational supplement. CLSI document M100-S25. Wayne, PA: CLSI; 2015.

26. Zar JH. Biostatistical analysis. 4a ed. Upper Saddle River: Prentice Hall; 1999.

27. Pinheiro RR, Gouveia AMG, Alves FSF, Haddad JPA. Aspectos epidemiológicos da caprinocultura cearense. Arq Bras Med Vet Zootec. 2000;52(5):1-14.

28. Andrade JSL, Azevedo SS, Teles JAA, Higino SSS, Azevedo EO. Ocorrência e fatores de risco associados à infecção por Corynebacterium pseudotuberculosis em caprinos e ovinos do semiárido paraibano. Pesqui Vet Bras. 2012;32(2):116-20.

29. Abreu SRO, Mota RA, Pinheiro Júnior JW, Rosinha GMS, Castro RS. Perfil de sensibilidade antimicrobiana in vitro de isolados de Corynebacterium pseudotuberculosis de caprinos e ovinos com linfadenite caseosa no sertão de Pernambuco, Brasil. Vet Zootec. 2008;15(3):502-9.

Recebido em: 21/11/2019

Aceito em: 17/07/2020 